\title{
Cesarean anesthetic management in a patient with Monoclonal Gammopathy. can we trust analytics?
}

\section{case report}

\section{Introduction}

Monoclonal gammopathies controlled by a heterogeneous group of disorders characterized by an uncontrolled proliferation of plasma cells that produce immunoglobulin molecules or fragments thereof, known as the monoclonal component. Within these, the Multiple Myeloma (MM) represents 13\% of hematological malignancies, its presentation during the fertile age is extremely unusual, and is characterized by the production of a monoclonal gammaglobulin, mainly responsible for its multiple conditions.

We expose 38-year-old woman case, who is diagnosed with Multiple Myeloma in her twenty-ninth week of gestation, where despite analytical values of platelets and coagulation within the limit of normal, after thromboelastometry, there is dysfunctionality in them.

\section{Clinical case}

38-year-old female patient, without known drug allergies. No medical-surgical history of interest. Pregnant in the third trimester of pregnancy, with good control and follow-up on an outpatient basis for obstetric consultations, presenting gestational hypothyroidism in treatment with Levothyroxine (Eutirox ${ }^{\circledR}$ ).

In the 29th week of gestation, the patient presented in the Emergency Department, due to a severe asthenia and drowsiness, accompanied by an analytical picture compatible with normochromic normocytic anemia ( $\mathrm{Hb} 8.5 \mathrm{~g} / \mathrm{dl}$, VCM $93 \mathrm{fl}$ ) and a slight elevation of plasma calcium $(11.1 \mathrm{mg} / \mathrm{dl})$. It is decided to enter charge of the gynecology service to complete study and interconsultation to the hematology service. The patient is diagnosed with MM, after an elevation of total proteins $(10.3 \mathrm{~g} / \mathrm{dl})$ with an $\mathrm{M}$ component in the gamma region in the proteinogram $(5.2 \mathrm{~g} / \mathrm{dl})$ and medullary plasmacyte in sternal puncture of $26 \%$. Elective caesarean section is scheduled to end pregnancy at week 34 , and to be able to start chemotherapy treatment.

The preoperative analysis showed values within the limit of normality, with a platelet count around $240 \times 103 / \mu \mathrm{L}$, and a coagulation without apparent alterations. Despite this, it was decided to perform a thromboelastometry test given the hematological pathology of the patient.

After performing the Thromboelastometry, a normal clotting time of clotting time was observed, accompanied by a maximum clot firmness of the maximum clott firmness of $42 \mathrm{~mm}$ in the EXTEM and $15 \mathrm{~mm}$ in the FIBTEM.

Given these results, caesarean section was performed under general anesthesia, with rapid sequence induction with rocuronium, without incidents. During the intervention, 1 "pool" of platelets was transfused, given the results obtained, indicative of a probable dysfunctionality in platelet aggregation.

The patient was subsequently transferred to the Resuscitation Unit, where after 24 hours of continuous monitoring and without presenting complications during her stay, she could be discharged.
Volume II Issue 3 - 2019

\author{
García Martínez J A, Rego Hermida P, \\ Guillamón Marín M J, Castañer Ramon-Llin \\ C \\ Resident of Anaesthesiology and Resuscitation Virgin University \\ Hospital of the Arrixaca, Spain
}

Correspondence: García Martínez J A, Resident of Anaesthesiology and Resuscitation Virgin University Hospital of the Arrixaca, Spain, Email josgamar007@gmail.com

Received: December 26, 2019 | Published: December 30, 2019

\section{Discussion}

Hematologic malignancies can be a challenge in the clinical practice of the anesthesiologist because of the alterations that these can produce at the blood level, sometimes insidiously as the case shows, and the difficulty that their management can infer during the perioperative time. ${ }^{1-5}$

The viscoelastic tests (TVE) have become a tool, which, at present, have been positioned as one of the most useful because of the "in vitro" information offered at the bedside. Thus facilitating an early and accurate interpretation/therapy. Laboratory tests only monitor the initial phase of coagulation, does not report the strength/fragility of the clot, being able to ignore clinically significant coagulopathies. On the other hand, when performed on platelet-poor plasma, they do not reflect the complex interaction between them and coagulation factors. TVE, on the other hand, use whole blood, so they better reflect the coagulation process and the interaction between the different blood components, allowing a functional determination of the status of the fastest and most complete coagulation. While it is true, it is not without important limitations (Figure 1).
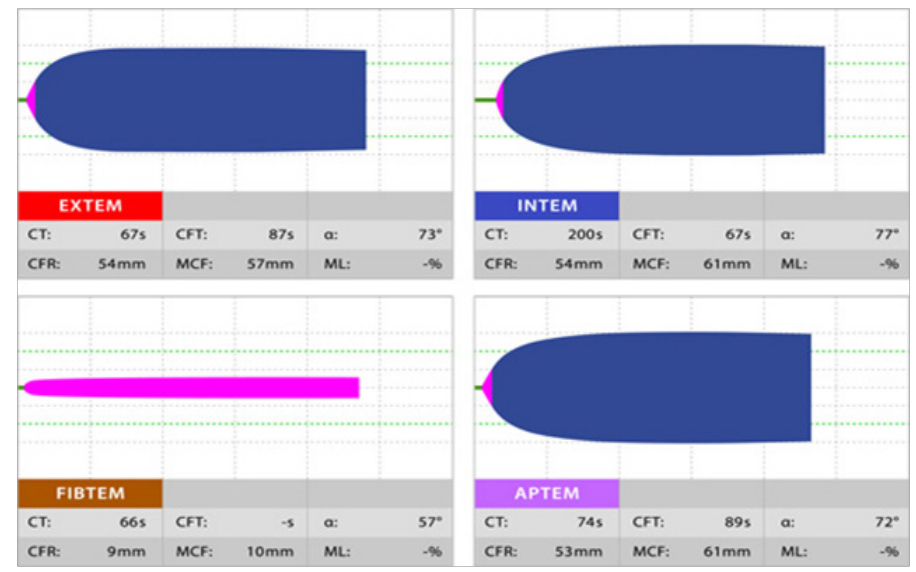


\section{Conclusion}

The appearance of a MM during pregnancy is extremely rare. Bone pain is the most frequent symptom due to the proliferation of tumor cells and the activation of osteoclasts with the consequent appearance of microfractures. The medullary displacement is accompanied by cytopenias, with anemia being the most frequent of them. Although the platelet value may be normal, there may be coagulation disorders due to platelet malfunctions, which may be coated with antibody or by the interaction of the $\mathrm{M}$ component with the coagulation factors I, II, V, VII and VIII.

The use of thromboelastometry in these cases becomes essential to assess the correct functioning of platelets. Finally, remember that MM can be accompanied in addition to impaired renal function and increased susceptibility to bacterial infections.

\section{References}

1. Bravo Garcia-Morato M, Padilla-Merlano B, Nozal P, et al. Laboratory guides for the diagnosis and monitoring of patients with monoclonal gammopathies. Spanish Clinical Magazine. 2016:216;128-134.

2. Hoffman M, Monroe DM. A cell-based model of hemostasis. Thromb Haemost. 2001;85(6):958-965.

3. Clinical guide of drugs that inhibit hemostasis and neuroaxial anesthesia. REAR. 2005;52:413-420.

4. Perez-Gomez F, Bover R. The new coagulation cascade and its possible influence on the difficult balance between thrombosis and hemorrhage. Rev Esp Cardiol. 2007;60(17)1217-1219.

5. Gutierrez Rico R.M. Hemostasis and thromboelastography in clinical situations. III CEEA Course: Intensive care, trauma, blood and transfusions. 2017. 